\title{
Sensitive vectorial optomechanical footprint of light in soft condensed matter
}

\author{
Mohamed El Ketara ${ }^{1,3}$, Hirokazu Kobayashi ${ }^{2,3}$ and Etienne Brasselet ${ }^{1}{ }^{1 凶}$
}

\begin{abstract}
Among the properties of light that dictate its mechanical effects, polarization has held a special place since the mechanical identification of the photon spin'. Nowadays, little surprise might be expected from the mechanical action of linearly polarized weakly focused (paraxial) beams on transparent and homogeneous dielectrics. Still, here we unveil vectorial optomechanical effects mediated by the material anisotropy and the longitudinal field component inherent to real-world beams ${ }^{2,3}$. Experimentally, this is demonstrated by using an elastic anisotropic medium prone to exhibit a sensitive and reversible effect, that is, a nematic liquid crystal, and our results are generalized to vector beams ${ }^{4}$. This represents an alternative to irreversible damaging approaches restricted to strongly non-paraxial fields ${ }^{5}$. The reported creation of multiple self-induced lenses from a single beam also open up topology assisted all-optical information routing strategies. Moreover, our findings point out the transverse internal optical energy flows (spin and orbital) ${ }^{6}$ as novel triggers to tailor structured optical nonlinearities.
\end{abstract}

From symmetry considerations, the ability of linearly polarized light to realign anisotropic material systems is an intuitive phenomenon and experimental demonstrations are plentiful. For instance, one could mention early works such as the optical reorientation of colour centres in salts ${ }^{7}$ or light-induced ordering in the isotropic $^{8}$ or ordered ${ }^{9}$ phase of liquid crystals in the 1970s. Of course, laser-induced alignment is not just the privilege of condensed matter systems-this has also been observed in molecular gases ${ }^{10,11}$, and the optomechanics of micro-objects ${ }^{12,13}$ and nano-objects ${ }^{14,15}$ under linearly polarized light mediated by anisotropy have also received a lot of attention. The experimental advances and the in-depth theoretical knowledge of paraxial wave optics acquired over the past decades thus leave little room today for highlighting new optomechanical effects in the simple case of a linearly polarized weakly focused (paraxial) beam propagating in an optically anisotropic medium.

Here we theoretically and experimentally unveil that a linearly polarized beam propagating along the optical axis of transparent and homogeneous uniaxial dielectrics can leave a vectorial mechanical footprint in the latter. The effect results from the optomechanical interference between the longitudinal and the transverse field components, which is inherent to real-world beams propagating in anisotropic media.

This effect is articulated by identifying the time-averaged optical radiation torque density $\boldsymbol{\Gamma}$ exerted in the bulk of an anisotropic slab that has its optical axis oriented along the $z$-axis. In dielectrics, $\boldsymbol{\Gamma}=\frac{1}{2} \operatorname{Re}\left(\mathbf{P}^{*} \times \mathbf{E}\right)$ where $\operatorname{Re}(\cdot)$ refers to the real part, $\mathbf{P}$ and $\mathbf{E}$ are the complex polarization density and electric field, respectively, and $(\cdot)^{*}$ refers to the complex conjugate. Recalling the constitutive relationship $\mathbf{P}=\epsilon_{0}(\overline{\bar{\epsilon}}-1) \mathbf{E}$, where $\epsilon_{0}$ is the vacuum permittivity and $\overline{\bar{\epsilon}}$ is the dielectric relative permittivity tensor, we have

$$
\Gamma=\frac{1}{2} \epsilon_{0} \epsilon_{a} \operatorname{Re}\left(E_{z}^{*} \mathbf{e}_{z} \times \mathbf{E}_{\perp}\right),
$$

where $\epsilon_{\mathrm{a}}=\epsilon_{\|}-\epsilon_{\perp}$ is the anisotropy of the dielectric permittivity tensor, $\epsilon_{\|}\left(\epsilon_{\perp}\right)$ is the dielectric relative permittivity parallel (perpendicular) to the optical axis and $\left(\mathbf{e}_{x}, \mathbf{e}_{y}, \mathbf{e}_{z}\right)$ is the unit basis of the Cartesian coordinate system. The torque density is thus mediated by the anisotropy of the material and results from the interference between the longitudinal $\left(E_{z}=\mathbf{E} \cdot \mathbf{e}_{z}\right)$ and the transverse $\left(\mathbf{E}_{\perp}=\mathbf{E}-E_{z} \mathbf{e}_{z}\right)$ parts of the field.

To exemplify the predicted effect, we choose the Gaussian beam as the incident field (labelled as '(i)') and we introduce the customary transverse field in cylindrical coordinates $(r, \phi, z)$, choosing a polarization state oriented along the $x$-axis. Omitting the propagation factor $\exp (-i \omega t+i k z)$, where $\omega$ is the angular frequency, $t$ is the time and $k$ is the wavevector, we have

$$
\mathbf{E}_{\perp}^{(\mathrm{i})}(r, z)=E_{0} \frac{w_{0}}{w(z)} \exp \left[-\frac{r^{2}}{w(z)^{2}}+i \frac{k r^{2}}{2 R(z)}-i \zeta(z)\right] \mathbf{e}_{x},
$$

where $E_{0}$ is the real maximal electric field amplitude, $w(z)=w_{0}\left(1+z^{2} / z_{0}^{2}\right)^{1 / 2}$ is the beam radius, $w_{0}$ is the beam waist radius, $z_{0}$ is the Rayleigh distance, $R(z)=z+z_{0}^{2} / z$ is the radius of curvature of the wavefront and $\zeta(z)=\arctan \left(z / z_{0}\right)$ is the Gouy phase. Reducing the total field to equation (2) - a purely transverse field-leads to a null torque; however, real-world beams do have a longitudinal component (as discussed in ref. ${ }^{2}$ for the isotropic case and more recently in ref. ${ }^{3}$ for uniaxial media) and a non-zero torque can thus be expected.

Here we consider an anisotropic slab with thickness $L$, whose mid-plane is located at $z$ and sandwiched between isotropic material with dielectric relative permittivity $\epsilon_{\perp}$ (this choice of refractive index matching is made for the sake of simplifying the analytical formulation of the problem as detailed in Methods), see Fig. 1a. In this case, the optical torque density can be calculated rigorously within the paraxial approximation, taking into account that the transverse field is altered over the course of its propagation in the anisotropic medium, which in turn modifies the longitudinal field ${ }^{16}$. As $\boldsymbol{\Gamma}$ thus depends on the position inside the anisotropic medium (see Extended Data 1), the typical behaviour is retrieved by looking at the longitudinal averaging, namely, $\left\langle\Gamma_{j}\right\rangle_{z}=\frac{1}{L} \int_{z-L / 2}^{z+L / 2} \Gamma\left(r, \phi, z^{\prime}\right) \cdot \mathbf{e}_{j} d z^{\prime}$, where $j=(x, y)$ (see Fig. 1b).

'Université de Bordeaux, CNRS, Laboratoire Ondes et Matière d'Aquitaine, Talence, France. ${ }^{2}$ School of System Engineering, Kochi University of Technology,

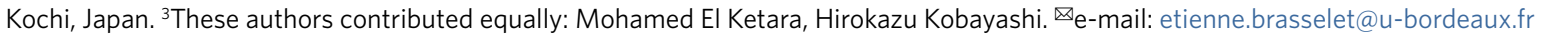


a

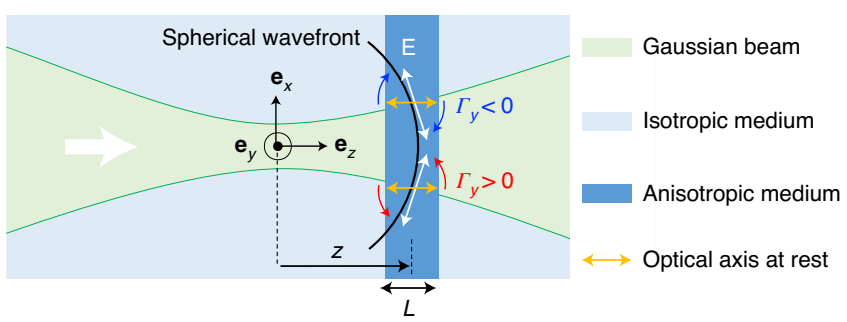

b

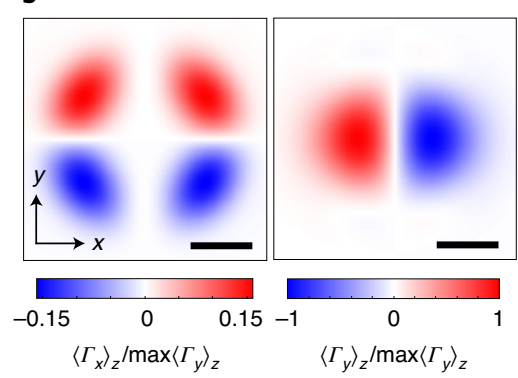

c

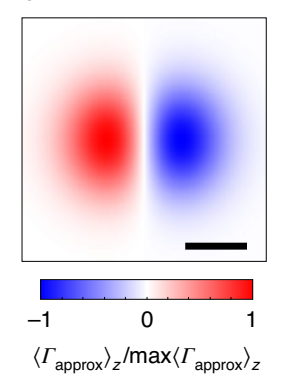

Fig. 1 | Vectorial optomechanics from a linearly polarized Gaussian beam. a, Light-matter interaction geometry. b, Calculated Cartesian components of the longitudinally averaged optical torque density. $\mathbf{c}$, Same as in $\mathbf{b}$ for the approximated expression given by equation (3), noting that $\Gamma_{\text {approx, } x}=0$. The calculations parameters are those of the experiment shown in Fig. 2b. Scale bar, $L / 2$.

It is noteworthy that the four-lobe pattern of $\Gamma_{x}$ originates from the interference between the two contracircular optical vortex fields with topological charges of \pm 2 generated by spin-orbit interactions.

A physical insight is gained by looking at the limit case when the effect of the anisotropy on the field can be discarded (that is, when $\epsilon_{\perp} / \epsilon_{\|} \rightarrow 1$ ), hence using the transverse field given by equation (2) and exploiting Maxwell equation $\nabla \cdot \overline{\bar{\epsilon}} \mathbf{E}=0$, which gives $E_{z}=\frac{i \epsilon_{\perp}}{k \epsilon_{\|}} \nabla_{\perp} \cdot \mathbf{E}_{\perp}$. After calculations, we have

$$
\boldsymbol{\Gamma}_{\mathrm{approx}}(r, \phi, z)=-\frac{\epsilon_{0} \epsilon_{a} \epsilon_{\perp}}{2 \epsilon_{\|}} \frac{r}{R(z)} \cos \phi\left|\mathrm{E}_{\perp}^{(\mathrm{i})}(\mathrm{r}, \mathrm{z})\right|^{2} \mathbf{e}_{\mathrm{y}},
$$

and comparison with the full calculation is made in Fig. $1 c$, which calls for a few comments. First, $\boldsymbol{\Gamma}_{\text {approx }} \cdot \mathbf{e}_{x}=0$ emphasizes that $\Gamma_{x}$ results from light-matter vectorial coupling mediated by the anisotropy, as is the multiple-lobe $(>2)$ modulation of $\Gamma_{y}$, which becomes prominent as the beam divergence angle increases (see Extended Data 1). Second, the crucial role of the wavefront curvature $1 / R$ arises; $E_{z} \neq 0$ is indeed a necessary condition-however non-sufficient - for $\boldsymbol{\Gamma} \neq 0$. Third, the torque reversal on either $z \rightarrow-z$ or $x \rightarrow-x$ can be well understood in the ray-optics region, which is typically defined as $|z|>z_{0}$. The field transversality with respect to the normal to the wavefront implies that the optical axis tends to realign along $\left(\epsilon_{a}>0\right)$ or perpendicular $\left(\epsilon_{a}<0\right)$ to the electric field (see Fig. 1a).

To unravel our predictions experimentally, we propose an elastic uniaxial medium. Liquid crystals, which are well-known for combining strong optical anisotropy with a spectacular orientational sensitivity to light fields ${ }^{17}$, are well suited for this purpose. We therefore prepare a nematic liquid crystal slab of $L=57 \mu \mathrm{m}$, which is sandwiched between two glass substrates to ensure that the director (that is, the unit vector $\mathbf{n}$ oriented along the local average orientation of the molecules) is always perpendicular to them at both ends of the slab while the optical axis in the bulk is free to realign under external stimuli (see Methods). The sample is irradiated at normal
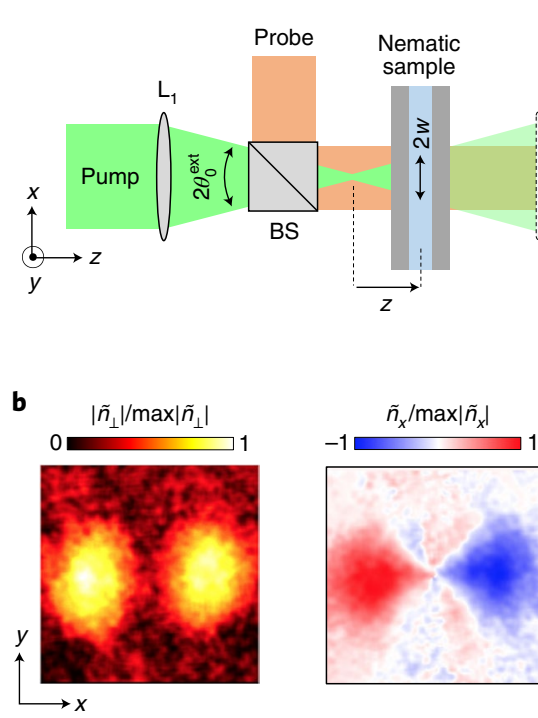

Probe module: analysing mechanical effects

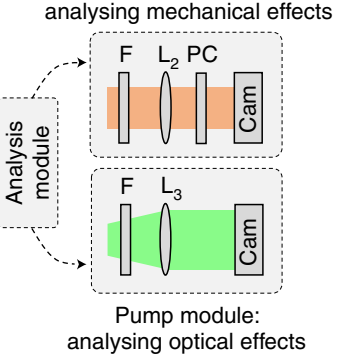

Fig. 2 | Vectorial optical structuring experiment under linearly polarized Gaussian beam. a, An illustrative set-up sketch (see Extended Data 2 for the full set-up). BS, beam-splitter; $F$, bandpass interferential filters; $L_{1}$, focusing lens for the input pump beam; $L_{2}$, imaging lens for the probe beam; $\mathrm{L}_{3}$, collimating lens for the output pump beam; $\mathrm{PC}$, polarization controller; Cam, camera. $\mathbf{b}$, Reconstructed spatial distribution of the director field in the mid-plane of the cell $\tilde{\mathbf{n}}_{\perp}=\left(\tilde{n}_{x}, \tilde{n}_{y}, 0\right)$, with $\max \left|\tilde{n}_{y}\right| / \max \left|\tilde{n}_{x}\right| \simeq 0.65$. Parameters: $\theta_{0}=\theta_{0}^{\text {ext }} / \epsilon_{\perp}^{1 / 2}=7.9^{\circ}$ is the internal Gaussian beam divergence, $\delta=2 \mathrm{w} / L=1.1$ and $P=96 \mathrm{~mW}$.

incidence by a Gaussian laser beam at $532 \mathrm{~nm}$ wavelength with incident power $P$ and linear polarization state oriented along the $x$-axis.

Vectorial optomechanical effects are analysed using the set-up depicted in Fig. 2a. Experimentally, the departure of the optical axis from the $z$-axis, $\mathbf{n}_{\perp}=\left(n_{x}, n_{y}, 0\right)$ is assessed from a polarization-resolved image of the mid-plane of the sample using a circularly polarized laser probe beam at $633 \mathrm{~nm}$ wavelength (see the 'probe module' box in Fig. $2 \mathrm{a})$. This is achieved by assuming non-twisted (along $z$ ) configuration of the optical axis and a small reorientation amplitude, which permits the fundamental longitudinal mode to be considered in isolation, namely, $\mathbf{n}_{\perp}=\tilde{n}_{x} \sin [\pi(Z+\xi) / L] \mathbf{e}_{x}+\tilde{n}_{y} \sin [\pi(Z+\xi) / L] \mathbf{e}_{y}$, where $Z=z-L / 2$ and $0<\xi<L$ (see Methods). Furthermore, optical structural changes are retrieved by monitoring the intensity distribution of the output pump beam whose collimation is adjusted in the absence of a detectable effect by setting $P \approx 1 \mathrm{~mW}$.

Typical results for the reconstructed director field are shown in Fig. $2 \mathrm{~b}$ at an aspect ratio of $\delta=2 w / L=1.1$ (see also Extended Data 3 for $\delta=2$ ). The comparison with theoretical predictions reported in Fig. $1 \mathrm{~b}$ requires a way to relate the optical torque density to its mechanical effects. This is achieved by noting that, locally, the mechanical response to the applied optical torque, $\mathbf{n}_{\perp} \neq 0$, should qualitatively follow $\mathbf{n}_{\perp} \propto \Gamma \times \mathbf{e}_{z}$, recalling that the unperturbed director field is $\mathbf{n}=\mathbf{e}_{z}$. We could therefore expect $\tilde{n}_{x} \propto\left\langle\Gamma_{y}\right\rangle_{z}$ and $\tilde{n}_{y} \propto-\left\langle\Gamma_{x}\right\rangle_{z}$, which brings a decent physical understanding of the phenomenon.

Complementary experiments show that the orientation of the double lens is adjustable by polarization means (see Fig. 3a, where the experimental normalized magnitude of the reconstructed optical axis perturbation is shown for an incident Gaussian beam with a linear polarization state oriented at an angle of $\beta= \pm 40^{\circ}$ from the $x$-axis). As the two lobes rotate together with the direction of the linear input polarization, one may wonder: what if the polarization plane instead rotates internally to the beam itself? 

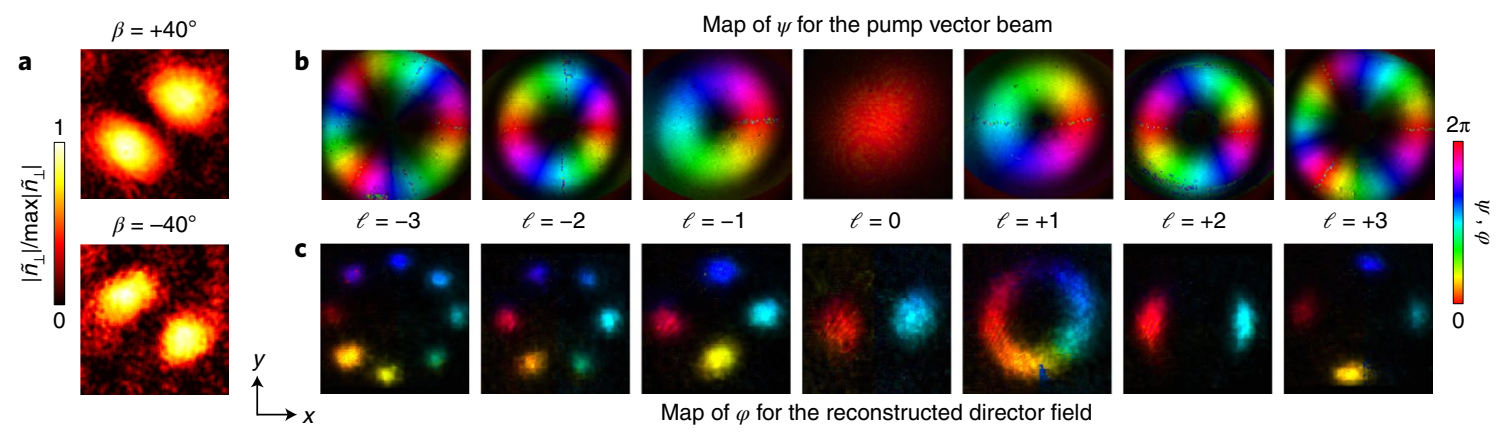

Fig. $3 \mid$ Vectorial control of vectorial optical structuring. a, A map of $\left|\tilde{\mathbf{n}}_{\perp}\right|$ for different orientation angles $(\beta)$ of the polarization plane. Parameters: $\theta_{0}=7.9^{\circ}$, $\delta=1.1$ and $P=96 \mathrm{~mW}$. b. Characterization of the incident optical vector field of order $\ell$. Luminance refers to the optical intensity, whereas the colour map refers to the polarization azimuth angle $(\psi)$. c. Characterization of $\tilde{\mathbf{n}}_{\perp}$. Luminance refers to $\left|\tilde{\mathbf{n}}_{\perp}\right|$ and the colour map refers to the in-plane orientation angle $\left(\tan \varphi=\mathrm{A}_{\mathrm{y}} / \mathrm{P}_{\mathrm{x}}\right)$. Parameters: $\theta_{0}=8.0^{\circ}, \delta=2.0$ and the optical power is set so that $\tilde{n}_{\perp} \approx 0.15-0.20$, namely, $P=669,544,464,250,383,464$ and $544 \mathrm{~mW}$ for $\ell$ ranging from -3 to +3 , respectively.

Such internal rotation of the polarization orientation is exactly what happens in vector beams endowed with a vector point singularity $^{18}$. The above question is therefore addressed by preparing linearly polarized pump beams with space-variant orientation of the polarization by using geometric phase optical elements ${ }^{4}$ (see Methods). Practically, the incident field is described by LaguerreGaussian vector beams whose electric field vector orientation varies azimuthally in a linear fashion in the $(x, y)$ plane, making a total rotation by an angle $2 \pi \ell$ per full turn around the $z$-axis, where the azimuthal index $\ell$ is an integer and $-3 \leq \ell \leq+3$, as shown in Fig. $3 \mathrm{~b}$ (see also Extended Data 4). The incident transverse field thus reads $\mathbf{E}_{\perp, \ell}^{(\mathrm{i})}(r, \phi, z)=E_{\perp, \ell}^{(\mathrm{i})}(r, z) \mathbf{e}_{\ell \phi}$ with $\mathbf{e}_{\ell \phi}=\cos \ell \phi \mathbf{e}_{\mathrm{x}}+\sin \ell \phi \mathbf{e}_{\mathrm{y}}$ and

$$
E_{\perp, \ell}^{(\mathrm{i})}(r, z)=\frac{2^{|\ell| / 2}}{\sqrt{\ell !}}\left(\frac{r}{w(z)}\right)^{|\ell|} e^{-i|\ell| \zeta(z)} E_{\perp}^{(\mathrm{i})}(r, z),
$$

where the prefactor ensures a constant optical power for all $\ell$ and $E_{\perp}^{(\mathrm{i})}(r, z)$ refers to the Gaussian amplitude given by equation (2). The case $\ell=0$ thus corresponds to the $x$-polarized Gaussian beam. The experimentally reconstructed maps of the director field perturbation are shown in Fig. $3 c$ and exhibit a $2(1-\ell)$-lobe vectorial pattern. A qualitative understanding of the observations can be grasped within the approximated framework previously discussed in the scalar case $(\ell=0)$. After calculations, we get

$$
\Gamma_{\text {approx }}^{(\ell)}=\frac{2^{|\ell|}}{\ell !}\left(\frac{r}{w(z)}\right)^{2|\ell|} \frac{\cos [(1-\ell) \phi]}{\cos \phi} \Gamma_{\text {approx }}^{(0)} \mathbf{e}_{\ell \phi+\frac{\pi}{2}},
$$

where $\Gamma_{\text {approx }}^{(0)}$ refers to the amplitude of equation (3). The $\cos [(1-\ell) \phi]$ term in the above equation indeed predicts $2|1-\ell|$ hot spots of optical axis perturbation. Moreover, $\mathbf{e}_{\ell \phi+\frac{\pi}{2}}$ explains the observation of azimuthally varying orientation of the anisotropic hot spots recalling the previously discussed qualitative expectation $\mathbf{n}_{\perp} \propto \Gamma \times \mathbf{e}_{z}$.

On noticing that the present light-matter interaction geometry has a long-standing history dating back to $1980^{\circ}$, which includes the specific roles of wavefront curvature ${ }^{19,20}$ and non-paraxiality ${ }^{21}$, our findings are surprising to say the least. Such a multipolar optomechanical response has not been reported so far. Moreover, apart from ref. ${ }^{21}$, all previously reported experimental attempts deal with optical power above the onset of the optical Fréedericksz instability, which refers to the fact that the initial equilibrium state $\mathbf{n}=\mathbf{e}_{z}$ is unstable above a threshold power $\left(P_{\text {th }}\right)$ within the paraxial wave optics treatment adopted since $1981^{22,23}$. Here, by contrast, the vectorial structural changes are observed at optical power below the customary $P_{\text {th }}$ (see Methods).
This naturally implies that there should be a reexamination of the concept of the Fréedericksz instability. On one hand, when recalling the role of the wavefront curvature emphasized by equation (3), we understand that the usual approach of placing the sample in the focal plane of the incident beam (where the curvature vanishes) prevents the observation of a vectorial-reoriented state for $P<P_{\mathrm{th}}$. On the other hand, studies purposely made with finite curvature nevertheless report a Gaussian-like reoriented state only above a power threshold, hence also indicating the occurence of optical Fréedericksz instability (see refs. ${ }^{19}$ and ${ }^{20}$, which deal with $1 / R \approx 0.02 \mathrm{~mm}^{-1}$ and $\delta \sim 0.7-0.8$ ). This suggests the existence of a transition between a scalar and a vectorial optomechanical regime as the curvature increases.

The above conjecture was tested with additional experiments performed under linear polarized scalar beams made at (almost) a constant beam diameter in the mid-plane of the sample and adjustable wavefront curvature, as shown in Fig. 4a (see Methods). By retrieving the optomechanical response using the pump module, we observe the gradual appearance of a nonlinear double-lens system aligned along the polarization direction (see Fig. 4b). The absence of a sharp transition between the two regimes indicates the role of noise. Specifically, here we are dealing with orientational fluctuations of thermal origin that are inherent to liquid crystal materials. Such orientational noise prevents the medium from reading the sign reversal of the longitudinal component of the light field as $x \rightarrow-x$ though enabling the usual optical Fréedericksz instability to occur as the curvature vanishes.

Furthermore, our experiments with vector beams bring the first experimental test of a previous numerical study ${ }^{24}$, although contradicting the numerical prediction of an azimuthally invariant perturbation magnitude instead of the experimentally observed string-of-pearls one. This pinpoints the crucial role of the longitudinal field, which is discarded in ref. ${ }^{24}$. Moreover, the unveiled azimuthally modulated optomechanical response echoes the tailored focal intensity landscapes theoretically discussed in the framework of tightly focused vector beams ${ }^{25}$. This again supports the importance of self-interference between the transverse and longitudinal components of light. Intriguingly, such a self-interference effect mediated by broken symmetry at interfaces has been previously conjectured to explain spiraling mass-transport driven by the orbital angular momentum of light ${ }^{26}$.

Exploiting the tensorial nature of the mechanical effects of light in uniaxial media, our findings emphasize the intrinsic vectorial nature real-world light beams, be they customarily considered as paraxial fields. The demonstration of multiple self-induced liquid crystal lenses from a single driving beam opens up topology assisted all-optical information routing strategies, recalling that 

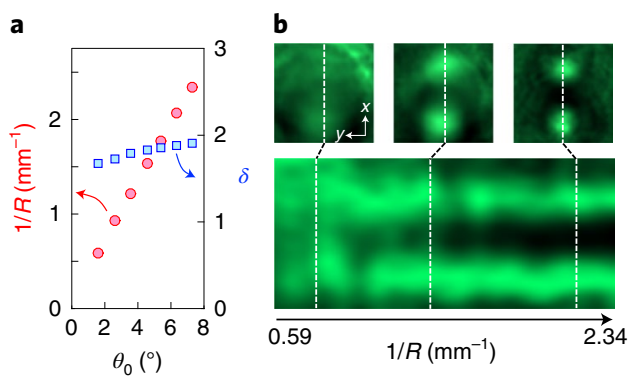

Fig. 4 | Wavefront curvature enabled vectorial optomechanics.

a, Measured relationships between $\theta_{0}$, the wavefront curvature of the incident light field $(1 / R)$ and the beam aspect ratio $(\delta)$ that refer to the data shown in panel $\mathbf{b}$. $\mathbf{b}$, Top row: intensity profiles of the output pump beam recorded using the pump beam analysis module shown in Fig. 2a, for three values of incident field curvature. For each image, the intensity is divided by that taken at $P=35 \mathrm{~mW}$ and then normalized to its own maximum for each value of the wavefront curvature. Bottom row: $x$-axis cross-section as a function of $1 / R$. The incident power is kept fixed at $P=230 \mathrm{~mW}$, which is smaller than $P_{\mathrm{th}}$, whichever value of $\theta_{0}$ considered.

liquid crystals are well-known for their capability to self-route the optical information via spatial solitons ${ }^{27}$. This adds to recent efforts to establish a soft-matter topological photonic toolbox ${ }^{28}$. We also anticipate a novel class of tailored structured optical nonlinearities from transverse internal optical energy flows of spin and/or orbital origin $^{6}$ whose mechanical effects have attracted a lot of experimental attention ${ }^{29-32}$. Indeed, by contrast to the present situation where the phenomenon vanishes in the focal plane of the driving field, either elliptically polarized beams or beams carrying orbital angular momentum can lead to non-zero optical torque density everywhere, including in the focal plane $e^{6}$. Earlier work using circularly polarized light far from the Rayleigh range ${ }^{33,34}$, is thus worth reconsidering. From an applied point of view, the sensitive detection of a cross-effect between the transverse and the longitudinal field components allows envisioning the development of novel 3D field reconstruction techniques that are not restricted to tight focusing conditions. Present study also contributes to the nonlinear optics of vector beams either in isotropic or anisotropic Kerr media (see, for instance, refs. ${ }^{35-37}$ ), in a context of ever increasing interest on both the fundamental and the technological aspects of vector light ${ }^{4}$.

\section{Online content}

Any methods, additional references, Nature Research reporting summaries, source data, extended data, supplementary information, acknowledgements, peer review information; details of author contributions and competing interests; and statements of data and code availability are available at https://doi.org/10.1038/ s41566-020-00726-2.

Received: 10 May 2020; Accepted: 21 October 2020;

Published online: 7 December 2020

\section{References}

1. Beth, R. A. Mechanical detection and measurement of the angular momentum of light. Phys. Rev. 50, 115-125 (1936).

2. Lax, M., Louisell, W. H. \& McKnight, W. B. From Maxwell to paraxial wave optics. Phys. Rev. A 11, 1365-1370 (1975).

3. Ciattoni, A., Crosignani, B. \& Di Porto, P. Vectorial theory of propagation in uniaxially anisotropic media. J. Opt. Soc. Am. A 18, 1656-1661 (2001).

4. Rosales-Guzmán, C., Ndagano, B. \& Forbes, A. A review of complex vector light fields and their applications. J. Opt. 20, 123001 (2018).

5. Hnatovsky, C., Shvedov, V., Krolikowski, W. \& Rode, A. Revealing local field structure of focused ultrashort pulses. Phys. Rev. Lett. 106, 123901 (2011).

6. Bekshaev, A., Bliokh, K. Y. \& Soskin, M. Internal flows and energy circulation in light beams. J. Opt. 13, 053001 (2011).
7. Blume, H., Bader, T. \& Luty, F. Bi-directional holographic information storage based on the optical reorientation of $\mathrm{F}_{\mathrm{A}}$ centers in KCl:Na. Opt. Commun. 12, 147-151 (1974).

8. Wong, G. K. \& Shen, Y. Optical-field-induced ordering in the isotropic phase of a nematic liquid crystal. Phys. Rev. Lett. 30, 895 (1973).

9. Zolot'ko, A. S., Kitaeva, V. F., Kroo, N., Sobolev, N. N. \& Csillag, L. The effect of an optical field on the nematic phase of the liquid crystal ocbp. JETP Lett. 32, 158-161 (1980).

10. Kim, W. \& Felker, P. M. Spectroscopy of pendular states in optical-field-aligned species. J. Chem. Phys. 104, 1147-1150 (1996).

11. Sakai, H. et al. Controlling the alignment of neutral molecules by a strong laser field. J. Chem. Phys. 110, 10235-10238 (1999).

12. Friese, M. E. J., Nieminen, T. A., Heckenberg, N. R. \& Rubinsztein-Dunlop, H. Optical alignment and spinning of laser-trapped microscopic particles. Nature 394, 348-350 (1998).

13. Higurashi, E., Sawada, R. \& Ito, T. Optically induced angular alignment of trapped birefringent micro-objects by linearly polarized light. Phys. Rev. E 59, 3676 (1999).

14. Tong, L., Miljkovic, V. D. \& Kall, M. Alignment, rotation, and spinning of single plasmonic nanoparticles and nanowires using polarization dependent optical forces. Nano Lett. 10, 268-273 (2010).

15. Liu, M., Zentgraf, T., Liu, Y., Bartal, G. \& Zhang, X. Light-driven nanoscale plasmonic motors. Nat. Nanotechnol. 5, 570-573 (2010).

16. Ciattoni, A., Cincotti, G. \& Palma, C. Propagation of cylindrically symmetric fields in uniaxial crystals. J. Opt. Soc. Am. A 19, 792-796 (2002).

17. Khoo, I. C. Nonlinear optics of liquid crystalline materials. Phys. Rep. 471, 221-267 (2009).

18. Freund, I. Polarization singularity indices in gaussian laser beams. Opt. Commun. 201, 251-270 (2002).

19. Santamato, E. \& Shen, Y. Field-curvature effect on the diffraction ring pattern of a laser beam dressed by spatial self-phase modulation in a nematic film. Opt. Lett. 9, 564-566 (1984).

20. Lucchetti, L., Suchand, S. \& Simoni, F. Fine structure in spatial self-phase modulation patterns: at a glance determination of the sign of optical nonlinearity in highly nonlinear films. J. Opt. A 11, 034002 (2009).

21. Lucchetti, L., Criante, L., Bracalente, F., Aieta, F. \& Simoni, F. Optical trapping induced by reorientational nonlocal effects in nematic liquid crystals. Phys. Rev. E 84, 021702 (2011).

22. Zel'dovich, B. Y., Tabiryan, N. V. \& Chilingaryan, Y. S. Fredericks transitions induced by light fields. Sov. Phys. JETP 54, 32-37 (1981).

23. Durbin, S., Arakelian, S. \& Shen, Y. Optical-field-induced birefringence and freedericksz transition in a nematic liquid crystal. Phys. Rev. Lett. 47, 1411 (1981)

24. Porenta, T., Ravnik, M. \& Zumer, S. Complex field-stabilised nematic defect structures in Laguerre-Gaussian optical tweezers. Soft Matter 8, 1865-1870 (2012).

25. Otte, E., Tekce, K. \& Denz, C. Tailored intensity landscapes by tight focusing of singular vector beams. Opt. Express 25, 20194-20201 (2017).

26. Ambrosio, A., Marrucci, L., Borbone, F., Roviello, A. \& Maddalena, P. Light-induced spiral mass transport in azo-polymer films under vortex-beam illumination. Nat. Commun. 3, 1-9 (2012).

27. Peccianti, M. \& Assanto, G. Nematicons. Phys. Rep. 516, 147-208 (2012).

28. Muševič, I. Integrated and topological liquid crystal photonics. Liq. Cryst. 41, 418-429 (2013).

29. Angelsky, O. et al. Orbital rotation without orbital angular momentum: mechanical action of the spin part of the internal energy flow in light beams. Opt. Express 20, 3563-3571 (2012).

30. Ruffner, D. B. \& Grier, D. G. Optical forces and torques in nonuniform beams of light. Phys. Rev. Lett. 108, 173602 (2012).

31. Antognozzi, M. et al. Direct measurements of the extraordinary optical momentum and transverse spin-dependent force using a nano-cantilever. Nat. Phys. 12, 731-735 (2016).

32. Svak, V. et al. Transverse spin forces and non-equilibrium particle dynamics in a circularly polarized vacuum optical trap. Nat. Commun. 9, 1-8 (2018).

33. Brasselet, E. Singular optical manipulation of birefringent elastic media using non-singular beams. Opt. Lett. 34, 3229-3231 (2009).

34. Brasselet, E. Singular optical reordering of liquid crystals using gaussian beams. J. Opt. 12, 124005 (2010).

35. Bouchard, F. et al. Polarization shaping for control of nonlinear propagation. Phys. Rev. Lett. 117, 233903 (2016).

36. Gu, B. et al. Varying polarization and spin angular momentum flux of radially polarized beams by anisotropic Kerr media. Opt. Lett. 41, 1566-1569 (2016).

37. Sakamoto, M. et al. Nonlinear propagation characteristics of a radially polarized beam in a uniaxially aligned dye-doped liquid crystal. J. Opt. Soc. Am. B 36, 3341-3347 (2019).

Publisher's note Springer Nature remains neutral with regard to jurisdictional claims in published maps and institutional affiliations.

(C) The Author(s), under exclusive licence to Springer Nature Limited 2020 


\section{Methods}

Propagation of a linearly polarized beam along the optical axis of uniaxial medium. The optical torque density $\boldsymbol{\Gamma}$, which accounts for the propagation effects, is calculated from analytical expressions within the paraxial framework. As light propagates along the optical axis, the input transverse field is altered due to the optical anisotropy, which in turn modifies the longitudinal field. Here we provide the expression of the total field inside the anisotropic medium following a previous theoretical work by Ciattoni and colleagues ${ }^{16}$. The light-matter interaction geometry is depicted in Fig. 1a. The mid-plane of the anisotropic slab of thickness $L$ is located at a distance $z$ from the incident field focal plane defining $z=0$. We thus consider an incident linearly polarized beam propagating along the $z$-axis and introduce the propagation distance inside the crystal, $0 \leq \xi \leq L$. According to equation (2), the input transverse field at $\xi=0$ is

$$
\mathbf{E}_{\perp}(r, \phi, Z)=E_{0} \frac{w_{0}}{w(Z)} \exp \left[-\frac{r^{2}}{w(Z)^{2}}+i \frac{k r^{2}}{2 R(Z)}-\mathrm{i} \zeta(Z)\right] \mathbf{e}_{x},
$$

where $Z=z-\frac{L}{2}$, recalling that we omit the unimportant pure phase propagating factor that is common to all field components inside of the crystal, namely, $\exp (-i \omega t+i k z)$, where $k=2 \pi n_{1} / \lambda_{\text {pump }}, \lambda_{\text {pump }}=532 \mathrm{~nm}$ is the pump wavelength in vacuum and $n_{\perp}=\epsilon_{\perp}^{1 / 2}\left(n_{\|}=\epsilon_{\|}^{1 / 2}\right)$ is the refractive index perpendicular (parallel) to the optical axis of the anisotropic medium. As light propagates inside of the anisotropic medium, the $x$ component of the field is altered whereas a non-zero $y$ component appears. Adapting the results of ref. ${ }^{16}$ to the present case, one gets

$$
\mathbf{E}_{\perp}(r, \phi, Z+\xi)=\left[A_{0}(r, Z, \xi)+A_{2}(r, Z, \xi) \cos (2 \phi)\right] \mathbf{e}_{x}+A_{2}(r, Z, \xi) \sin (2 \phi) \mathbf{e}_{y},
$$

where

$$
\begin{gathered}
A_{0}(r, Z, \xi)=A(Z)\left[\alpha_{\perp}(Z, \xi) e^{-\alpha_{\perp}(Z, \xi) r^{2}}+\alpha_{\|}(Z, \xi) e^{-\alpha_{\|}(Z, \xi) r^{2}}\right], \\
A_{2}(r, Z, \xi)=A(Z)\left[\left(\alpha_{\perp}(Z, \xi)+\frac{1}{r^{2}}\right) e^{-\alpha_{\perp}(Z, \xi) r^{2}}-\left(\alpha_{\|}(Z, \xi)+\frac{1}{r^{2}}\right) e^{-\alpha_{\|}(Z, \xi) r^{2}}\right],
\end{gathered}
$$

and

$$
\begin{gathered}
\alpha_{\perp}(Z, \xi)=\left[w_{0} w(Z) e^{\mathrm{i} \zeta(\mathrm{Z})}+i \frac{2 \xi}{k}\right]^{-1} \\
\alpha_{\|}(Z, \xi)=\left[w_{0} w(Z) e^{\mathrm{i} \zeta(\mathrm{Z})}+i \frac{2 \epsilon_{\perp} \xi}{k \epsilon_{\|}}\right]^{-1}, \\
A(Z)=\frac{1}{2} w_{0}^{2} E_{0} .
\end{gathered}
$$

The longitudinal component of the field is then calculated from $E_{z}=\frac{i \varepsilon_{\perp}}{k \epsilon_{\|}} \nabla_{\perp} \cdot \mathbf{E}_{\perp}$, which, after calculations, gives

$$
E_{z}(r, \phi, Z+\xi)=-i A(Z) \frac{4 \epsilon_{\perp}}{k \epsilon_{\|}} \alpha_{\perp}^{2}(Z, \xi) r e^{-\alpha_{\perp}(Z, \xi) r^{2}} \cos \phi
$$

Here we recall that both the transverse and the longitudinal components are necessary to evaluate the optical torque density, see equation (1). By inserting equations (7) and (13) into equation (1), we get

$$
\begin{aligned}
& \Gamma_{x}=-\frac{1}{2} \epsilon_{0} \epsilon_{a} \operatorname{Re}\left(E_{z}^{*} E_{\perp, y}\right), \\
& \Gamma_{y}=+\frac{1}{2} \epsilon_{0} \epsilon_{a} \operatorname{Re}\left(E_{z}^{*} E_{\perp, x}\right),
\end{aligned}
$$

where the notation $\mathbf{E}_{\perp}=E_{\perp, x} \mathbf{e}_{x}+E_{\perp, y} \mathbf{e}_{y}$ is used. We can confirm that the above expressions simplify to equation (3) in the limit $\epsilon_{\perp} / \epsilon_{\|} \rightarrow 1$. The calculated evolution of the transverse distribution of the $\Gamma_{x}$ and $\Gamma_{y}$ inside of the crystal are shown in Extended Data 1 for $\delta=2 w(z) / L=1.5$, which is a representative value of the reported experiments that belong to the typical range $1<\delta<2$. We also consider two values for $\theta_{0}=\lambda_{\text {pump }} /\left(\pi n_{\perp} w_{0}\right)$, namely, $\theta_{0}=1^{\circ}$ and $\theta_{0}=10^{\circ}$. This allows for appreciation of how the optical torque density components are modified as light propagates inside of the anisotropic medium, starting from $\Gamma_{y} \equiv \Gamma_{\text {approx }}$ and $\Gamma_{x} \equiv 0$ at $\xi=0$ (whichever value of $\theta_{0}$ ), where $\Gamma_{\text {approx }}$ is given by equation (3). In particular, the relative weight of $\Gamma_{x}$ to the magnitude of the total optical torque density vanish as the beam divergence tends to zero. Indeed, $\max \left(\left.\Gamma_{x}\right|_{\xi=L}\right)$ is typically one-thousandth of $\max \left(\left.\Gamma_{y}\right|_{\xi=L}\right)$ for $\theta_{0}=1^{\circ}$, whereas $\max \left(\left.\Gamma_{x}\right|_{\xi=L}\right) \approx \max \left(\left.\Gamma_{y}\right|_{\xi=L}\right)$ for $\theta_{0}=10^{\circ}$. The magnitude of the optical torque density itself also vanishes as the beam divergence tends to zero. Indeed, $\left.\left.\max \left(\left.\Gamma_{y}\right|_{\xi=L}\right)\right|_{\theta_{0}=1^{\circ}} \approx 10^{-3} \max \left(\left.\Gamma_{y}\right|_{\xi=L}\right)\right|_{\theta_{0}=10^{\circ}}$.

Detailed experimental set-up. The detailed experimental set-up is depicted in Extended Data 2. Hereafter we provide details about the preparation of the pump beam ( $532 \mathrm{~nm}$ wavelength), the preparation of the probe beam $(633 \mathrm{~nm}$ wavelength), the nematic sample and the analysis modules for both the pump and the probe beams.

Pump beam preparation. A spatial filter based on a $200-\mu \mathrm{m}$-diameter pinhole placed in the focal plane of an afocal pair of converging lenses is used to prepare the incident Gaussian beam whose polarization state at the output of the laser cavity is homogeneous and linear. The cleaned beam is then expanded by a $\times 5$ commercial beam expander and the beam is focused either by using a single lens (as is the case for the data shown in Fig. 2b, Fig. 3 and Extended Data 3) or by using a more elaborate system comprising a pair of lenses, as shown in Extended Data 2. The latter option allows for control of $\theta_{0}$ while keeping the beam diameter almost constant at $2 w$ in the mid-plane of the cell, which is evaluated at $\exp (-2)$ of the maximal optical intensity. This is made by varying the distance $D$ between the two lenses as the first lens is displaced along the $z$-axis. Such a protocol allows for a series of experiments to be performed using $\theta_{0}$ as a control parameter at an almost fixed aspect ratio $\delta$ (see Fig. 4a). When vector beams are used (see Fig. 3), a vortex plate made of a space-variant half-wave plate is placed before the lens that is used to focus the pump beam. The orientation angle of the optical axis of the vortex plate is given by $\psi=\ell \phi / 2$, which provides a vector beam with a linear polarization state that is oriented along $\mathbf{e}_{\ell \phi}=\cos \ell \phi \mathbf{e}_{\mathrm{x}}+\sin \ell \phi \mathbf{e}_{\mathrm{y}}$ for an input beam polarized along the $x$-axis. The transverse field expression given by equation (4) - which refers to the coherent superposition of two fundamental LaguerreGaussian beams: one being right-handed circularly polarized and having an azimuthal index $\ell$ and the other being left-handed circularly polarized and having an azimuthal index $-\ell$ ) - is experimentally tested from the radial dependence of the intensity profiles, as summarized in Extended Data 4, where the solid curves refer to the Laguerre-Gaussian lineshapes. Finally, $P$ is measured between the beam-splitter and the nematic sample, accounting for $9 \%$ transmission losses (that is, half of the measured input/output transmission losses of $18 \%$ that are attributed to a combination of Fresnel reflections and light scattering from the nematic slab).

Probe beam preparation. The probe beam from a He-Ne laser is expanded into a collimated beam with a diameter of a few millimetres using an afocal pair of lenses. Its polarization state is prepared to be circularly polarized after its reflection by a non-polarizing beam-splitter. A double rotating diffuser ${ }^{38}$ is placed in the course of the beam before it impinges onto the beam-splitter to reduce disturbances of a speckle origin, hence improving the quality of the images of the sample using the probe light. Still, this technique is only applied here to produce Extended Data $5 \mathrm{~b}$, whereas the spatially resolved polarimetric analysis used to reconstruct the vectorial structure of the director field presented in Fig. 2b, Fig. 3 and Extended Data 3 are all made without the diffuser add-on.

Nematic sample. We use the nematic liquid crystal E7 (Merck), with refractive indices $n_{\|}=1.756$ and $n_{\perp}=1.528$ at $25^{\circ} \mathrm{C}$ and $532 \mathrm{~nm}$ wavelength. The $1-\mathrm{mm}$-thick borosilicate glass substrates - with a refractive index of 1.52 at $532 \mathrm{~nm}$ wavelength-are treated with cetyl-trimethyl-ammonium-bromide surfactant that provides uniform molecular orientation of the liquid crystal along the $z$-axis at rest. In the presence of a light-induced mechanical realignment of the director (that is, the unit vector $\mathbf{n}$ oriented along the local average orientation of the molecules, which defines the local orientation of the optical axis) in the bulk, the director always remains perpendicular to the substrates at both ends of the nematic slab. The nematic sample has $L=57 \mu \mathrm{m}$. The nematic layer is placed after the focal plane of the focused pump beam, which is located in the bulk of the glass substrate (as sketched in Extended Data 2).

Probe beam analysis. The output probe beam is used to image the mid-plane of the sample using the imaging lens. In practice, the distance between the lens and the camera is $D^{\prime \prime}=35 \mathrm{~cm}$, and the distance between the sample and the lens is slightly larger than the focal length of the lens. The polarization analysis of the image allows extracting a quantitative information regarding the light-induced optical axis reorientation in the nematic slab.

Pump beam analysis. The output pump beam is deflected into a separate optical arm owing to a beam-splitter and a mirror (see Extended Data 2), and its analysis is performed according to the following protocol. First, the incident optical power is reduced enough to prevent substantial optomechanical effects from taking place, and a lens is placed to collimate the beam. The use of different pump beam divergence angles (see Fig. 4) requires to adapt the position of the collimating lens to the value of $\theta_{0}$. Consequently, the diameter of the collimated beam varies with $\theta_{0}$. To minimize the latter variation, we use a set of three collimating lenses by decreasing its focal length as $\theta_{0}$ increases. This gives a collimated beam diameter that ranges between $1.4 \mathrm{~mm}$ and $2.2 \mathrm{~mm}$. Further pump beam images collected by the camera are then resized to have identical initial observation conditions in the 
absence of any detectable effects. When the mechanical effects of light are large enough, self-focusing effects are observed. In the experiment reported in Fig. 4 , which was made at fixed $P$, the $D^{\prime}$ between the collimating lens and the camera is chosen to minimize the size of the observed hot spots. In practice, this corresponds to $10 \mathrm{~cm} \lesssim D^{\prime} \lesssim 50 \mathrm{~cm}$

Reconstructed perturbation of the director field. The perturbation of the director field $\tilde{\mathbf{n}}_{\perp}=\tilde{n}_{x} \mathbf{e}_{x}+\tilde{n}_{y} \mathbf{e}_{y}$ is reconstructed experimentally using the probe beam analysis module, exploiting the polarimetric analysis of the image of the mid-plane of the sample (see Extended Data 2). It relies on the description of the transverse part $\mathbf{n}_{\perp}=\left(n_{x}, n_{y}, 0\right)$ of the liquid crystal director field $\mathbf{n}=\left(n_{x}, n_{y}, n_{z}\right)$ by non-twisted deformations, assuming that only the fundamental longitudinal orientational mode is excited. The latter assumption is satisfied for small reorientation angles, as in our experiments where the maximal reorientation angle with respect to the $z$-axis is estimated to satisfy $\vartheta<10^{\circ}$ in all cases, namely, $n_{j}(x, y)=\tilde{n}_{j}(x, y) \sin (\pi \xi / \mathrm{L})$ with $j=(x, y)$. Within this framework, the transverse director field can be rewritten as a function of two angles, $\vartheta=\tilde{n}_{\perp}=\left(\tilde{n}_{x}^{2}+\tilde{n}_{y}^{2}\right)^{1 / 2}$ and $\varphi$ defined as $\tan \varphi=\tilde{n}_{x} / \tilde{n}_{y}$, according to

$$
\begin{aligned}
& \tilde{n}_{x}(x, y)=\vartheta(x, y) \cos \varphi(\mathrm{x}, \mathrm{y}), \\
& \tilde{n}_{y}(x, y)=\vartheta(x, y) \sin \varphi(\mathrm{x}, \mathrm{y}) .
\end{aligned}
$$

The experimental determination of $\tilde{\mathbf{n}}_{\perp}$ thus requires the pair of angles $(\vartheta, \varphi)$ to be determined. This is achieved by polarimetric Stokes imaging of the sample formed, see the probe analysis module in Extended Data 2, which requires at least four independent polarization measurements ${ }^{39}$. In practice, we measure the spatial distribution of the three reduced Stokes parameters $\left(s_{1}, s_{2}, s_{3}\right)^{40}$. On one hand, using a left-handed circularly polarized incident probe beam (that is, the electric field vector rotates from $x$ to $y$ in a plane perpendicular to the $z$-axis) and noting that we take care that the probe beam has a maximal birefringent phase retardation $\Delta$ not larger than $\pi / 2$, the azimuth of the output polarization ellipse is oriented at $+45^{\circ}$ from $\mathbf{n}_{\perp}$. We therefore get

$$
\varphi(x, y)=\frac{1}{2} \arctan \left[\frac{s_{2}(x, y)}{s_{1}(x, y)}\right]-\frac{\pi}{4} .
$$

On the other hand, the relationship between $\vartheta$ and $\Delta$ is obtained from the optics of uniaxial media, which gives

$$
\Delta=\frac{2 \pi}{\lambda_{\text {probe }}} \int_{0}^{L}\left(\left[\frac{\epsilon_{\|} \epsilon_{\perp}}{\epsilon_{\|} \cos ^{2}[\vartheta \sin (\pi \xi / \mathrm{L})]+\epsilon_{\perp} \sin ^{2}[\vartheta \sin (\pi \xi / \mathrm{L}))}\right]^{1 / 2}-\epsilon_{\perp}^{1 / 2}\right) \mathrm{d} \xi
$$

where $\lambda_{\text {probe }}=633 \mathrm{~nm}$ is the wavelength of the probe beam. Recalling that $\vartheta^{2} \ll 1$, we therefore get $\Delta=\left[\pi \epsilon_{a} \epsilon_{\perp}^{1 / 2} /\left(2 \epsilon_{\|}\right)\right]\left(L / \lambda_{\text {probe }}\right) \vartheta^{2}$, and, as $\Delta<\pi / 2$ and $s_{3}=\cos \Delta$, we eventually obtain

$$
\vartheta(x, y)=\left[\frac{2 \epsilon_{\|} \lambda_{\text {probe }}}{\pi \epsilon_{a} \epsilon_{\perp}^{1 / 2} L} \arccos s_{3}(x, y)\right]^{1 / 2}
$$

Determining the power threshold of the optical Fréedericksz instability. As stated in the main text, the reported experiments are all carried out below the power threshold of the optical Fréedericksz instability, above which the unperturbed director field corresponds to an unstable equilibrium state with the customary paraxial wave optics theoretical treatment. To clarify these points on experimental grounds, we chose $\theta_{0}=1.7^{\circ}$, at which vectorial structuring was not observed (see Fig. 4), which we interpret as the signature of the unavoidable existence of orientational fluctuations of thermal origin. The usual optical Fréedericksz instability is therefore expected in this case. This was tested by retrieving the optomechanical response of the sample as a function of $P$ (see Extended Data 5, which rely on observations using the pump (panel a) and the probe (panel b) analysis modules). From Extended Data Fig. 5a, axisymmetric output intensity profiles are observed and a sudden change occurs at $P=P_{\text {th }}=233 \pm 1 \mathrm{~mW}$, above which self-focusing effects appear and further develop as $P$ increases, with the remarkable appearance of self-phase modulation ring patterns. This qualitatively matches with the early observations associated with the optical Fréedericksz instability ${ }^{9,41}$, as well as quantitatively with regards to the threshold power, whose analytical expression is available neglecting the elastic anisotropy ${ }^{42}$,

$$
P_{\text {th }}=\frac{\pi^{3} c K \epsilon_{\|}}{8 \epsilon_{\perp}^{1 / 2} \epsilon_{a}}\left(\delta+\frac{2 \sqrt{2}}{\pi}\right)^{2}
$$

where $c$ is the speed of light in vacuum and $K$ is the effective Frank elastic constant. Indeed, experimental parameters imply $K=11.3 \mathrm{pN}$, which matches, within $1 \%$, to the average of the tabulated elastic constants ${ }^{43}$. Furthermore, the probe beam images of the sample obtained between crossed circular polarizers shown in Extended Data 5b match with previous works that all mention the rise of on-axis Gaussian-like reorientation profiles when $P>P_{\text {th }}$, with a reorientation magnitude that monotonously increases with $P$ (refs. ${ }^{9,41}$ ). Note that the formation of a on-axis doughnut intensity profile as the power increases is associated with the fact that Extended Data $5 \mathrm{~b}$ refers to crossed circular polarization imaging, which implies that the observed probe intensity profiles satisfy $I(x, y) \propto \sin ^{2}[\Delta(x, y) / 2]$, where $\Delta(x, y)$ refers to the transverse distribution of the birefringent phase retardation experienced by the probe beam. The formation of rings are thus expected as $P$ increases as $\Delta$ increases with $P$. Finally, noting that $P_{\text {th }}$ increases with $\delta$, see equation (21), the series of experiments reported in Fig. 4 are all carried out at $P<P_{\mathrm{th}}$. In addition, the experimental data shown in Fig. $2 \mathrm{~b}$ refers to $P \approx 0.7 P_{\mathrm{th}}$, that of Extended Data 3 refers to $P \approx 0.8 P_{\text {th }}$.

\section{Data availability}

The data that support the findings of this study are available on request from the corresponding author.

\section{References}

38. Lowenthal, S. \& Joyeux, D. Speckle removal by a slowly moving diffuser associated with a motionless diffuser. J. Opt. Soc. A 61, 847-851 (1971).

39. Vedel, M., Breugnot, S. \& Lechocinski, N. Full stokes polarization imaging camera. SPIE Proc. 8160, 81600X (2011).

40. Born, M. \& Wolf, E. Principles of Optics (Pergamon, 2005).

41. Durbin, S., Arakelian, S. \& Shen, Y. Laser-induced diffraction rings from a nematic-liquid-crystal film. Opt. Lett. 6, 411-413 (1981).

42. Zolot'ko, A. S. et al. Light-induced second-order phase transition in a spatially bounded region of a nematic liquid crystal. JETP Lett. 36, 80-84 (1982).

43. Strömer, J., Raynes, E. \& Brown, C. Study of elastic constant ratios in nematic liquid crystals. Appl. Phys. Lett. 88, 051915 (2006).

44. Brasselet, E., Lherbier, A. \& Dubé, L. J. Transverse nonlocal effects in optical reorientation of nematic liquid crystals. J. Opt. Soc. Am. B 23, 36-44 (2006).

\section{Acknowledgements}

This work has been partially funded by the Research Foundation for Opto-Science and Technology and JSPS KAKENHI (grant no. 20K05364)

\section{Author contributions}

E.B. conceived and supervised the project. All authors contributed to the experimental and theoretical results. E.B. wrote the manuscript with contributions from other authors.

\section{Competing interests}

The authors declare no competing interests. 
(a)
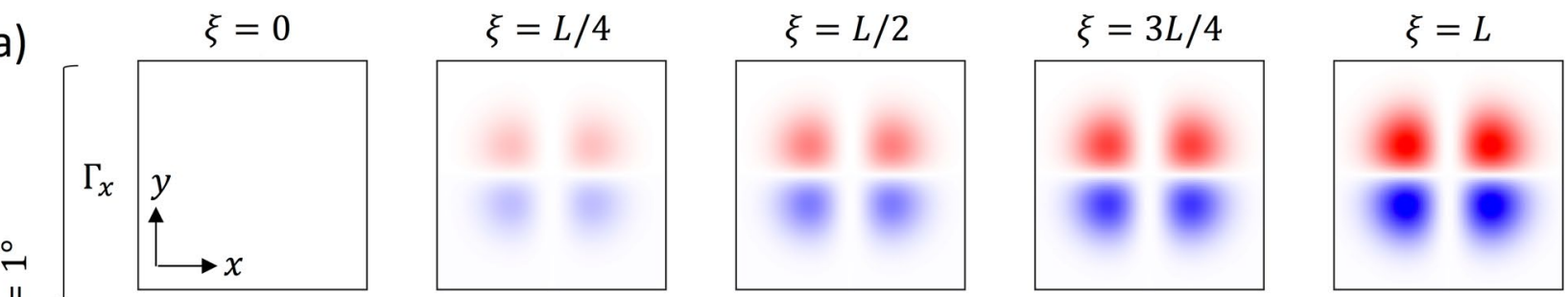

1

II
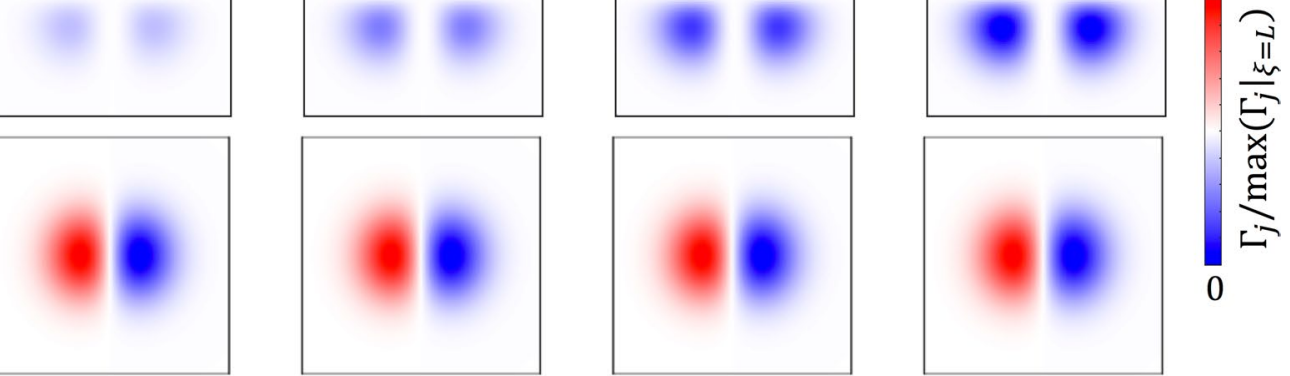

(b)
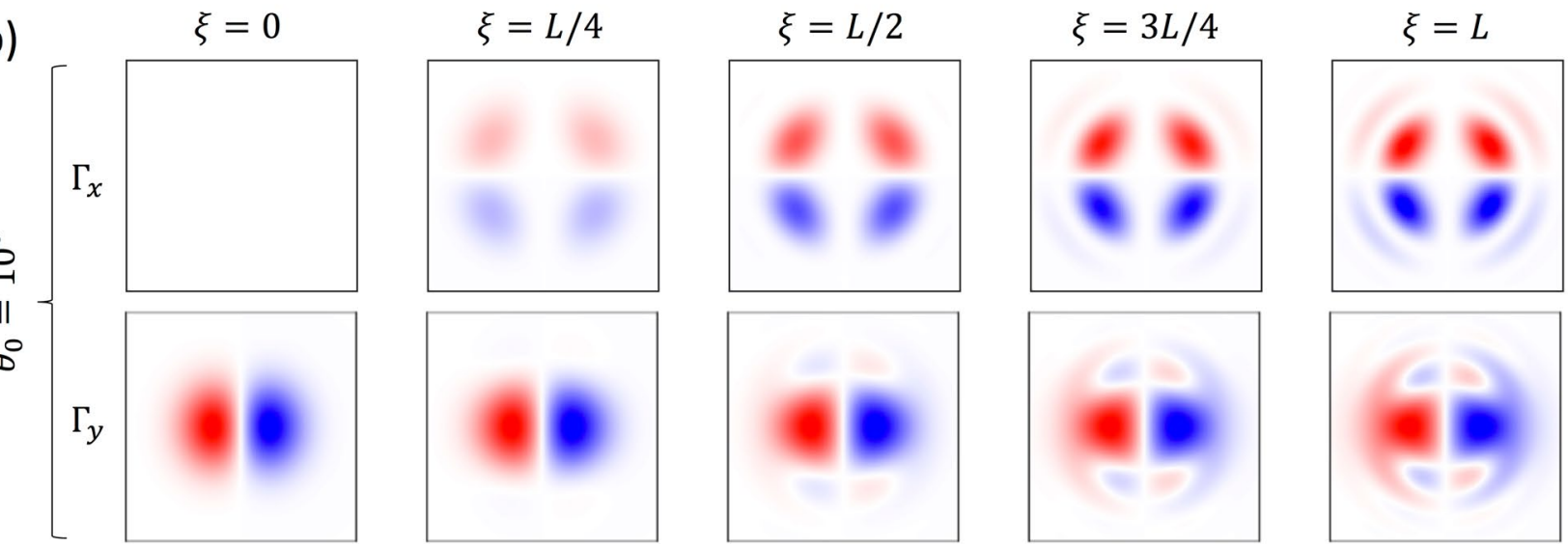

1

Extended Data Fig. 1 | Calculated transverse optical torque density inside the unperturbed anisotropic slab. Calculated transverse spatial profiles of the normalized optical torque density Cartesian components $\Gamma_{x}$ and $\Gamma_{y}$ for an incident Gaussian field having a linear polarization state oriented along the $x$ axis and propagating along the $z$ axis, where $0 \leq \xi \leq L$ refers to the propagation distance along the optical axis of the crystal from its input facet that defines $\xi=0$. Two values of the internal Gaussian beam divergence angle are considered: $\theta_{0}=1^{\circ}(\mathrm{a})$ and $\theta_{0}=10^{\circ}(\mathrm{b})$. Each box is centered on $(x, y)=(0,0)$ and covers an area $\delta L \times \delta L$ where $\delta=2 w(z) / L$. Each plot is normalized to the maximal value of the plot at $\xi=L$ on the same row and $\max \left(\left.\Gamma_{x}\right|_{\xi=L}\right)=1.2 \times 10^{-3} \max \left(\left.\Gamma_{y}\right|_{\xi=L}\right)$ for $\theta_{0}=1^{\circ}$ whereas $\max \left(\left.\Gamma_{x}\right|_{\xi=L}\right)=0.60 \max \left(\left.\Gamma_{y}\right|_{\xi=L}\right)$ for $\theta_{0}=10^{\circ}$. Parameters: $\delta=1.5, L=57 \mu \mathrm{m}, n_{\|}=1.756$ and $n_{\perp}=$ 1.528. See Methods for details. 


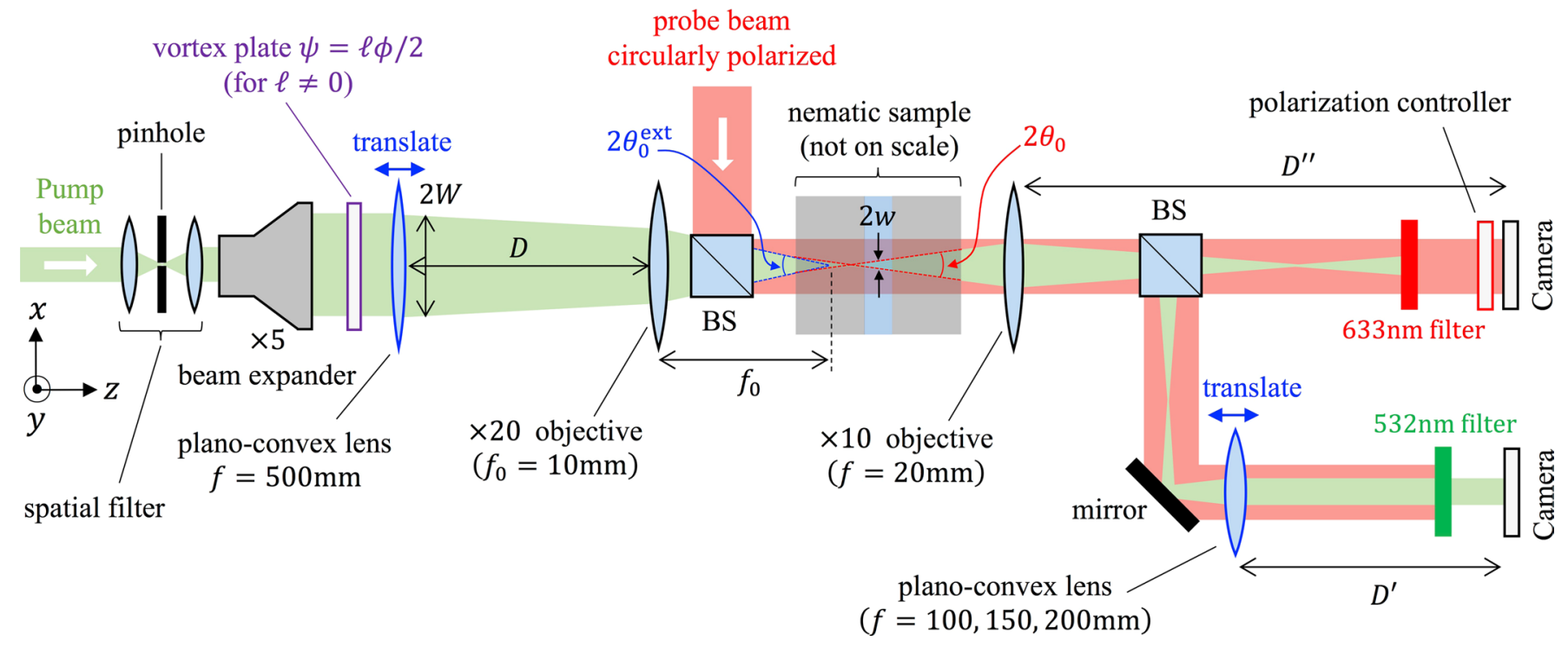

Extended Data Fig. 2 | Detailed experimental setup. Extended version of the illustrative experimental setup shown in Fig. 2(a). BS: non-polarizing beamsplitter for the probe beam. Note that Fig. 2(a) only summarizes the main instrumental ingredients used in practice. Namely, the lens L2 in Fig. 2 (a) is an imaging lens for the probe beam in order to observe the director field at the mid-plane of the liquid crystal sample, which corresponds to the $10 \times$ objective lens as shown here. Also, the lens L3 in Fig. 2(a) refers to a collimating lens for the pump beam, which corresponds to a lens system made of a 10 $\times$ objective lens followed by a plano-convex lens $(f=100,150,200 \mathrm{~mm})$ as shown here. See Methods for details. 

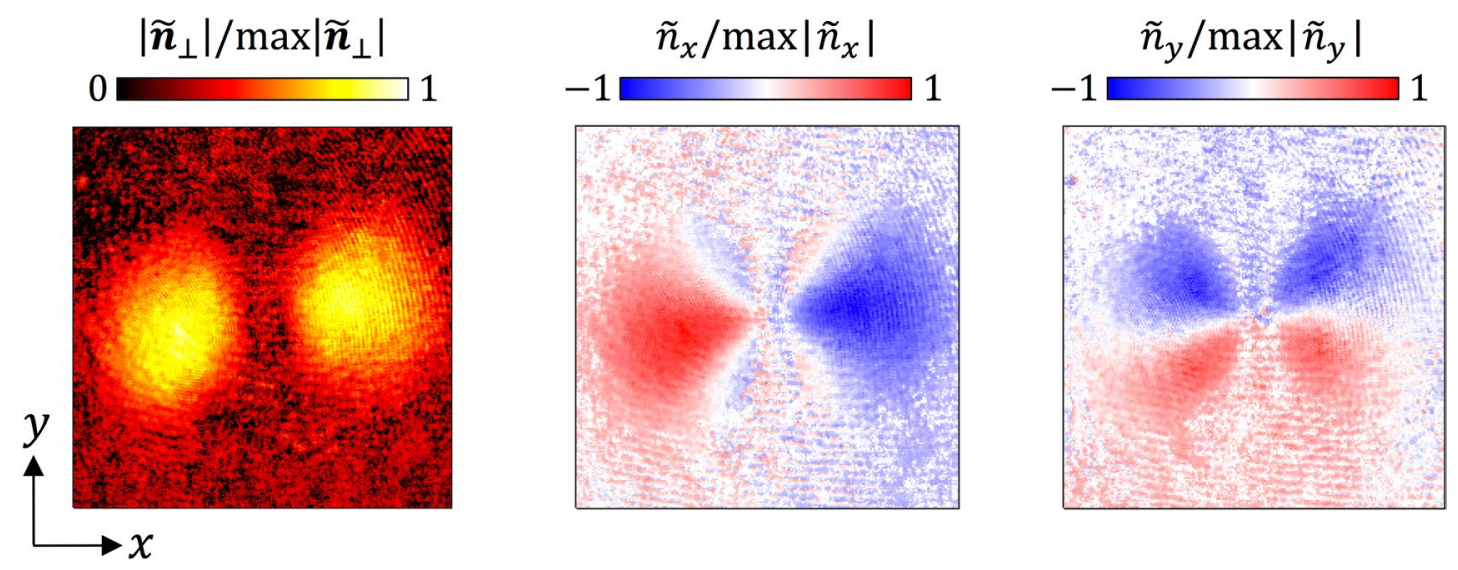

Extended Data Fig. 3 | Experimental reconstruction of the director field perturbation for incident light with uniform linear polarization state.

Reconstructed spatial distribution of the director field in the mid-plane of the cell $\tilde{\mathbf{n}}_{\perp}=\left(\tilde{n}_{x}, \tilde{n}_{y}, 0\right)$ as in Fig. 2 (b) but for another set of parameters: $\theta_{0}=8$. $0^{\circ}, \delta=2.0$ and $P=250 \mathrm{~mW}$. Here $\max \left|\tilde{n}_{y}\right| / \max \left|\tilde{n}_{x}\right| \simeq 0.88$. This demonstrates the robustness of the effect versus the diameter of the beam. We notice that the required power to reach the same magnitude for the material response increases with $\delta$ whereas choosing $\delta>1$ facilitates the observations. The latter point can be understood from the fact that the nonlocal character of the nematic response is strengthened as $\delta$ decreases ${ }^{44}$. 

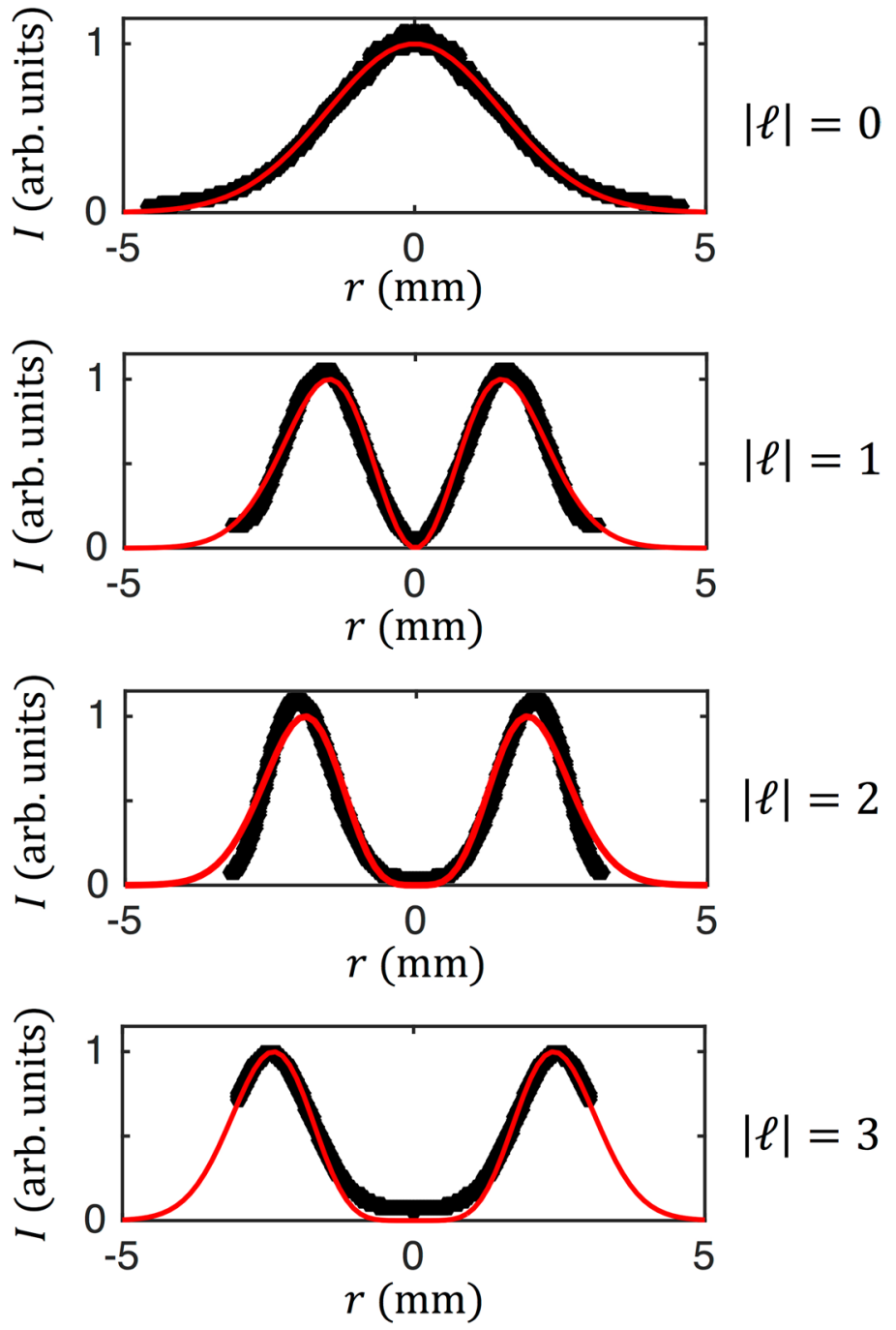

Extended Data Fig. 4 | Transverse intensity profiles of the incident vector beams. Testing the Laguerre-Gaussian lineshapes of the prepared vector beams. Markers: azimuthally-averaged radial intensity profiles measured just before the focusing lens, a $\times 10$ microscope objective with a numerical aperture $N A=0.4$. Solid curves: best fit using the Eq. (4). 


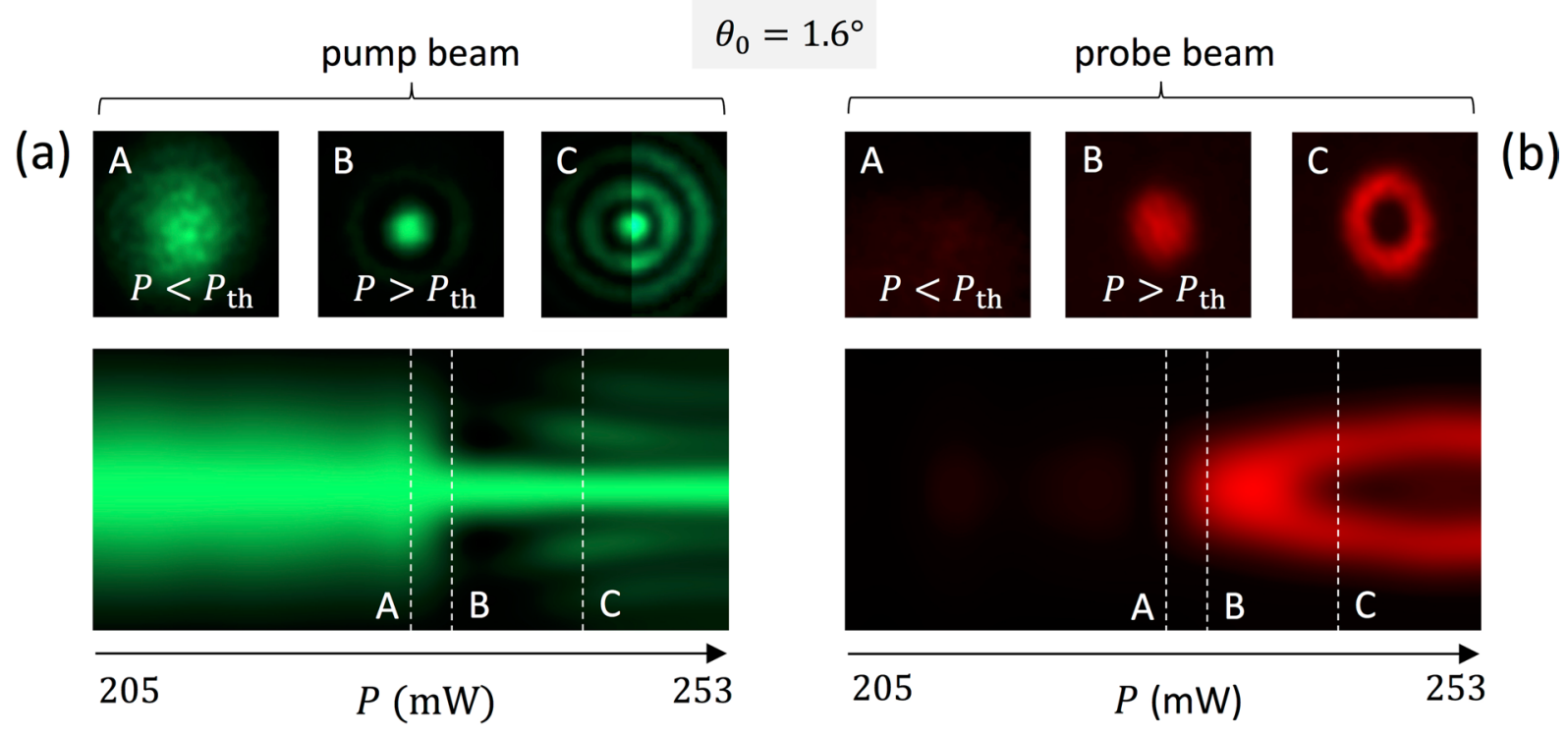

Extended Data Fig. 5 | Pump and probe optical characterization of the customary light-induced Fréedericksz instability. Experimental determination of the customary optical Fréedericksz instability for $\theta_{0}=1.6^{\circ}$ and $\delta=1.7$ from the pump beam analysis (a) and from probe beam analysis (b). Set of selected power values that corresponds to the transverse intensity profiles shown in the top row: $P_{\mathrm{A}}=231 \mathrm{~mW}, P_{\mathrm{B}}=235 \mathrm{~mW}, P_{\mathrm{C}}=246 \mathrm{~mW}$ while $P_{\text {th }}=233 \pm 1 \mathrm{~mW}$. In panel (a), case $C$, the luminance has been enhanced on the right side of the image in order to better visualize the self-phase modulation intensity rings. 